\title{
DBET: Demand Based Energy efficient Topology for MANETs
}

\author{
${ }^{1}$ Pawar Priyanka, ${ }^{2}$ Auti Sarika, ${ }^{3}$ Jadhav Roshana, ${ }^{4}$ Dighe Snehal \\ ${ }^{1,2,3,4}$ Computer Department SGOI College of Engineering, Belhe, Pune.
}

\begin{abstract}
Energy efficient topology in Ad-hoc networks can be achieved mainly in two different ways. In the first method, network maintains a small number of nodes to form a connected backbone and the remaining nodes sleep to conserve energy. This method is effective for low traffic networks. Energy efficiency in the second method is achieved by power control technique. This technique is effective in high traffic conditions. The first method is not effective in high traffic conditions. Similarly, the second method is not effective in low traffic networks. So, in this paper we propose a Demand Based Energy efficient Topology (DBET) to reduce the energy consumption for mobile ad hoc network, by dynamically adjusting the topology for various network traffic conditions. We have simulated our proposed protocol DBET by using AODV [8] as routing protocol in network simulator ns2.33 [1] and compared with AODV and SPAN [3]. The simulation studies revealed that the proposed scheme perform better in terms of energy, delay, and delivery ratio.in this paper newly we are implementing the security factor while sending the packets. For that we using RSA algorithm.
\end{abstract}

\section{Introduction}

Mobile Ad-hoc Networks (MANETs) are self-organizing, self-configuring and infrastructure-less multi-hop wireless net-works, where each node communicates with other nodes directly or indirectly through intermediate nodes without any infrastructure. Such temporary networks can be used in battle-fields, disaster areas, military applications, mining operations and robot data acquisition. Besides these characteristics they present challenges like limited energy, dynamic topology, low bandwidth and security. The description of the arrangement of the MANETs, called topology, is usually temporary or dynamically changed with time.

Energy conserving is one of the challenges because of limited battery resource. The techniques which are used to reduce the initial topology of network to save the energy and increase the lifetime of network, with the preservence of network connectivity, called topology control techniques. Various techniques, in network layer, are proposed in the literature to conserve energy. These techniques can be clas-sified mainly into two categories: by controlling the number of nodes with the smaller link cost. In the first method a small number of nodes awake to maintain the network connectivity and remaining nodes go into sleep state to conserve energy. This method is effective in low traffic conditions, because the power consumption to keep nodes awake dominates the power consumption in data transfer. In the second method, topology is controlled by keeping lesser cost links in the network.

This method is effective in high data traffic because power consumption in data transfer dominates the power required to keep nodes awake. We combine the advantages of these two techniques to dynamically adjust network topology for various network traffic conditions.

In this paper, we present a demand based energy efficient topology (DBET) that dynamically adjust network topology for various network traffic conditions. We have simulated our proposed protocol DBET by using AODV [8] as routing protocol using network simulator ns2.33 [1] and compared with AODV and AODV with SPAN [3]. The simulation studies revealed that the proposed scheme perform better in terms of energy, delay, and delivery ratio. In general network topology is controlled by keeping small number of nodes awake as in the first technique. The proposed DBET keeps more number of nodes along the bulk data transfer path to conserve energy by keeping low link cost as in the second technique.

The rest of the paper is organized as follows: the next section provides a brief review of related studies. The third section gives the design details of proposed DBET. Integration issues of DBET with routing protocol is discussed in the forth section. Simulation results along with discussions are provided in the section 5. The last and final section concludes the paper with same pointers to future research direction.

\section{Related Work}

We briefly describe various techniques related to our work topology control. Different topologies have been proposed in the literature to reduce the energy consumption. These methods can be classified into centralized controlling and distributed computing methods. Ideally, for mobile ad hoc network a topology should be computed and maintained in distributed, asynchronous, and localized manner.

$\mathrm{Li}$ and Wan [6] described a distributed protocol to construct a minimum power topology and developed an algorithm which directly find a path whose length is within a constant factor of the shortest path. The length of the path is measured in term of energy consumption. This proposed algorithm used only local information. 
A topology based on minimum spanning tree, called local-ized minimum spanning tree (LMST) was proposed by Li et al. [5]. It is a localized distributed protocol with the following properties: (1) the protocol generates a strongly connected communication graph; (2) the degree of any node is at most six, and (3) the topology can be made symmetric by removing asymmetric links without impairing connectivity.

A simple localized distributed topological control algorithm (XTC) [12] is proposed by Wattenhpfer et al. Initially each node $u$ computes a total order $\left(<_{u}\right)$ over all its neighbors in the network graph $G$ with respect to decreasing link quality, such as signal attenuation, and packet arrival rate. Then, each node start exchanges the neighbor order information among all neighbors. At the later point, each node locally selects those neighboring nodes which will form its neighborhood in the resulting topology control graph, based on the previously exchanged neighbor order informations. It covers three main advantages: 1) It does not assume the network graph to be a Unit Disk Graph, 2) it works on weighted network graphs, and 3) it does not require availability of the node position information. Authors improved by adding node mobility and extended XTC for mobile network [7].

An energy efficient dynamic path is maintained to send data from source to destination for MANET is proposed in Sheu, Tu, and Hsu [10]. Due to mobility existing paths may not be energy efficient. So, each node in a data path dynamically updates the path by adjusting its transmission power. Each node in the networks determines its power for data transmission and control packets transmission according to the received beacon messages from its neighbors. In dynamic path optimization technique protocols dynamically select energy efficient path as per the requirement of dynamic topological changes in the network [4].

Another class of topology control protocols based on the $k$-nearest neighbors graph ( $k$-NNG). In $k$ NNG every node is connected to its $k$ closest neighbors. The Local Information No Topology protocol (LINT) [9] try to keep the number of neighbors of a node within a low and high threshold centered around an optimal value. But the optimal value is not characterized. So the estimation of the number of neighbors can be inaccurate and connectivity is not guaranteed. The $k$-Neighbors ( $k$-NEIGH) [2] protocol keeps the number of neighbors of a node equal to, or slightly below, a given value $k$. It connects each node with $k$-closest neighbor, instant of require the knowledge of all the neighbors. The communication graph that result is made symmetric by removing asymmetric edges, which has $k$-upper bound neighbors. A characterization of the critical neighbor numbers is discussed by Xue and Kumar [13].

Another way of reducing the power consumption is by using efficient energy path for transmitting packets. These methods choose smaller edges in their path to reduce transmission energy. Minimum energy consumption per packet can be achieved by choosing the optimal energy consumed path from source to destination. However, this technique does not take the nodes' energy capacity into consideration. So some nodes may exhaust their power since energy consumption is not fair among the nodes in the network. There for the network lift-time decrease [11].

\section{Demand Based Energy Efficient Topology}

In this section, we present a demand based energy efficient topology (DBET) for mobile ad hoc network, which dynam-ically changes the topology according to the network traffic requirements. Initially we compute a small set of nodes, which form a connected backbone, while the other nodes are put off to conserve energy. These connected backbone is used for routing the packets under low network load. When there is a bulk data transfer between a pair of nodes, the topology dynamically changes along the path between these nodes by power control and route optimize technique to minimize the power consumption.

The proposed DBET can be divided into four phases. The first phase selects a small set of nodes that constitutes a inde-pendent set of the network. The second phase is responsible for electing more nodes to ensure that the selected nodes form a connected backbone. Remaining nodes go to sleep to conserve energy. Active node withdraw process is implement in the third phase to remove redundant nodes in each region. To improve the performance along the high traffic path we use the route optimization with power control technique in the fourth phase. In this technique, we change topology dynamically to connect more nodes, around the routing path to minimize the total power consumption.

\section{A. Phase I: Independent set formation}

The first phase selects a minimal set of nodes that constitute a minimal independent set of a connected backbone of the network. This selection is done in a distributed and localized manner using neighbor information available with the network layer. Let $n_{i}$ be the total number of nodes surrounding a node $i$ and let $n_{a i}$ be the number of additional nodes among these neighbors, which are connected, if node $i$ becomes a coordinator to the forward packets. The following heuristic is used in this phase: 
- Stability factor (denoted by $S$ ): Nodes that are relatively more stable as compared to the others in the localities are given more preference. The node's stability is measured as the ratio of number of link failures $\left(f_{i}\right)$ and new connection established $\left(c_{i}\right)$ per unit time to the total number of nodes surrounding that node $\left(n_{i}\right)$. Therefore,

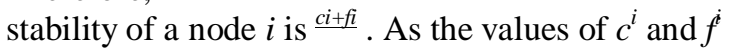

$n_{i}$

increase, the stability of the node decreases.

- Utility factor (denoted by U): Nodes that have higher number of neighbors without an active neighbor are given more preference. This heuristic is derived from the fact that such nodes, if elected, can help a larger number of other nodes, which can then be put to sleep state. Thus,

the utility factor $U^{i}$ of a node $i$ is calculate as ${ }^{n i-n a i}$.

$n_{i}$

- Energy factor (denoted by E): Nodes that have higher amounts of percentage remaining power are given more preference over others to be elected as active nodes. This

introduces fairness in the protocol by ensuring proper rotation in the selection of active nodes. Let $E_{0 i}$ denote the initial node's energy and $E_{t i}$ be the amount of energy

of a node at time $t$. So the energy factor $E_{i}$ of the node

$i$ is calculate as $E 0 i-E t i$.

${ }^{E} 0_{i}$

Thus, the above discussion suggests that the coordinator selection factor for phase - I can be the sum of all these factors

$$
\begin{aligned}
& C i=S i+U i+E i={ }^{c i+f i}+{ }^{n i-n a} i+{ }^{E} 0 i^{-E t} i \\
& n_{i}
\end{aligned} n_{i} \text { (1) } \quad{ }^{E} 0_{i}
$$

Only nodes that do not have an active node in their neighbor-hood are allowed to participate in the election. Announcement contention occurs when multiple nodes discover the lack of an active node, and all decide to become active nodes. We resolve the contention by delaying the announcement with randomized back off delay, which is proportional to the extent to which the node satisfies the heuristics. The selected nodes forms an independent set of a connecting backbone of the network. Selected active nodes $h_{t}{ }^{2} h_{r}{ }^{2}$ go back to sleep after they have used up a fixed percentage of their power to ensure fairness and $d^{4} \quad$ allow other nodes to become active.

\section{B. Phase II: Connecting the Independent Set}

Nodes selected in the first phase are not connected. This is because there is only one active node in a given locality. In this phase more nodes are elected to ensure that the selected nodes form a connected network.

All nodes that have two or more active nodes as neighbors, which are not connected directly or through one or two active nodes, are eligible to become active in this phase. Preference is given to the nodes satisfying the following criteria:

- $\quad$ Nodes having higher amount of remaining energy.

- $\quad$ Nodes having higher stability. This can be measured similar to the one used in the first phase.

- $\quad$ Nodes having more number of active nodes in the

1-hop neighborhood.

The stability and energy factors of this phase is very much similar with $1^{\text {st }}$ phase. But the utility factor is depends upon the $1^{\text {st }}$ phase's black active nodes. Let $n_{b i}$ be the number of active nodes of the $1^{s t}$ phase in $1-h o p$ neighborhood of a node $i$. If nodes with high $n_{b i}$ become the coordinators in this phase, fewer coordinators in total may be needed in order to make sure every node can talk to a coordinator. Thus a node with a high $n_{b i}$ should volunteer more quickly than one with smaller value. Thus, the coordinator selection factor for $2^{\text {nd }}$ phase is the sum of all these factors

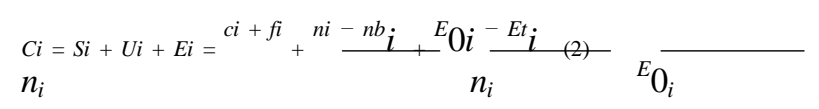


The contention if any is also resolved using the back off mechanism like in the first phase.

\section{Phase III: Coordinator Withdraw}

Every active node periodically checks if it should goto sleep state or not. The need for a node to be an active may also cease to exist due to the dynamics of the system. More explicitly, this may happen due to one of the following reasons.

- If first phase active nodes may move into a region that already has another first phase active node so that the region now has more than one first phase active nodes. These active nodes recognize this situation and one of them withdraws.

- If the withdrawal of a first phase active node may mean that the second phase active nodes in the locality no longer serve their purpose and hence withdraw.

In the above scenarios the respective active nodes withdraw, as their need no longer exists. However, when an active node withdraws by virtue of completion of its quota of time it needs to be awake until another node is elected in the locality.

The first three phases of proposed DBET and two phases of SPAN are similar. However DBET have the following advantages over SPAN.

- The proposed DBET uses only 1-hop neighbor informa-tion to create an energy efficient conneacted backbone network, but the SPAN use 2-hop neighbor information.

- The SPAN consider only energy and utility factor for co-ordinator's announcement phase, but the proposed DBET also consider the stability factor for improving the stabil-ity of the backbone.

- The SPAN uses same utility factor for all nodes, but proposed DBET's utility factors $\left(U_{A}\right.$ and $\left.U_{B}\right)$ are depend upon node's type. Thus, the total number of coordinators (active nodes) are fewer then the SPAN.

\section{Phase IV: Local route customization with Power control technique}

The energy consumption per data packet form source to destination is high when each node uses full transmission power. This can be reduced by chooses a lower energy cost path. The minimum transmission power $P_{t}(d)=a d^{k}+c$ is required to send data to a node at a distance $d$, where $2<k<4$ and for some constants $a$ and $c$. The receiving

power $P_{r}=P_{t} G_{t} G_{r} \quad=P_{t d^{\frac{K}{4}}}$ by surface reflection

model, where $h_{t}, G_{t}, h_{r}$, and $G_{r}$ are respectively antenna height and gain of sending and receiving nodes [10]. The

actual power $\xi^{l, J}=K^{\underline{P t}}+X$, required for sending data from

$P_{r}$

Our proposedDBET uses this power optimization technique locally along the routing path to minimize the energy consumption during the transmission.

\section{Integrating Dbet With Routing Protocol}

The proposed DBET can be integrated with any routing protocol. In this section, we discusses the process of integration with AODV. In our approach all control packets and data packets are transmit on low traffic path with full transmission power and data packets on high traffic path with minimum required energy.

Route discovery: Route discovery uses route cost in place of hop count as route metric. We use the notation $\delta_{I, J}$ denotes the cost of least cost path from the node $I$ to the node $J$.

When a source node $S$ wants to find a route to a des-tination $D$, it broadcasts the route request packet (RREQ) to its neighbors. The route request packet contains the least route cost from source node $S$, which is initially zero. An intermediate node $J$ receiving the route request packet from another intermediate node $I$, it calculates the cost of the path form node $S$ to nodes $J$ as $\delta_{S, I}+\xi_{I, J}$. The node $J$ update its routing table if the calculated cost is less than the cost in its routing table and forward the route request packet to its neighbors with updated cost. In order to avoid another cost update, node $J$ waits for the time (propositional to the cost to $\delta_{S, J}$ ) before forwarding. 
When a destination node $D$ receives first route request packet (RREQ), it calculates the route cost and update its routing table. It waits for a fixed time interval to receive more route request packets and find the least cost route among them. The node $D$ unicast a route reply packet (RREP) back to its neighbor from which it received the least cost route. The neighbor nodes unicast RREP towards the source node $S$.

Local route customization: As we discussed earlier due to the dynamic nature of the network new node may come closer to existing path, which may reduce the existing route cost, if it participates in forwarding the data.

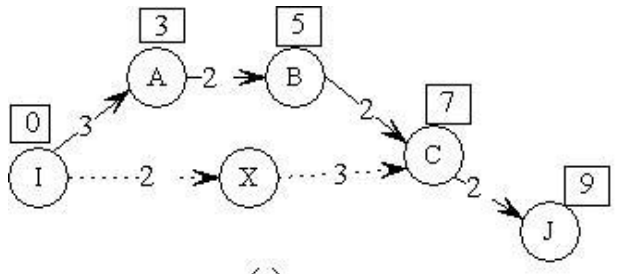

(a)

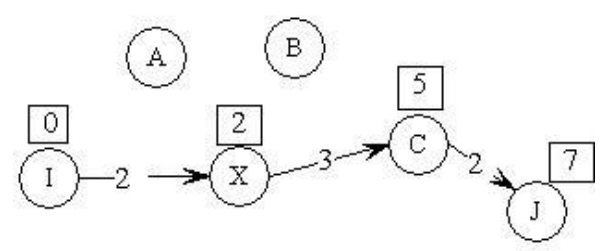

(b)

Fig. 2. Local path customization.

Let consider the example network given in the Fig. 2(a) with the existing path cost from the node $I$ to the node $J$ is 9 units. If a node is in data transmission path, it sends the

< Source address, Destination address, Route cost from

source to itself $>$ as a piggyback with periodic hello messages in full transmission path. After receive the hello messages from the node $I$ and the node $C$, along with piggyback information, node $X$ calculate the link $\operatorname{cost} \xi_{I, X}$ and $\xi_{C, X}$ and checks whether it can participate in the ongoing data transfer.

\section{Conclusions}

In this paper, we proposed a demand based energy efficient topology that dynamically adjusts its topology for various network traffic conditions. We have simulated our proposed protocol DBET and compared with AODV and AODV with SPAN. The simulation studies revealed that the proposed scheme perform better in terms of energy, delay, and delivery ratio. It would be interesting to investigate the use of direc-tional antenna to further reduce the energy consumption.

\section{References}

[1] The network simulator - ns-2.33. http://www.isi.edu/nsnam/ns.

[2] Douglas M. Blough, Mauro Leoncini, Giovanni Resta, and Paolo Santi. The k-neighbors approach to interference bounded and symmetric topology control in ad hoc networks. IEEE Transactions on Mobile Computing, 5(9):1267-1282, 2006.

[3] Benjie Chen, Kyle Jamieson, Hari Balakrishnan, and Robert Morris. Span: An energy-efficient coordination algorithm for topology mainte-nance in ad hoc wireless networks. ACM Wireless Networks, 8:85-96, September 2001

[4] H. P. Gupta and S. V. Rao. Pclr: Power control-based locally customize routing for manet. In Proc. IEEE International Conference on RF and Signal Processing Systems, pages 632-637, 2010.

[5] N. Li, J.C. Hou, and L. Sha. Design and analysis of an mst-based topology control algorithm. In INFOCOM 2003. Twenty-Second Annual Joint Conference of the IEEE Computer and Communications. IEEE Societies, pages 1702 - 1712, mar. 2003.

[6] Xiang-Yang Li and Peng-Jun Wan. Constructing minimum energy mobile wireless networks. SIGMOBILE Mob. Comput. Commun. Rev., 5(4):55-67, 2001. 